\title{
Quantitative Convergence for Cerebral Processing of Information within the Geomagnetic Environment
}

\author{
Mandy A. Scott ${ }^{1}$, Michael A. Persinger ${ }^{1,2^{*}}$ \\ ${ }^{1}$ Behavioural Neuroscience Programs, Laurentian University, Sudbury, Canada; ${ }^{2}$ Biomolecular Sciences Programs, Laurentian Uni- \\ versity, Sudbury, Canada. \\ Email: "mpersinger@lauerntian.ca
}

Received June $16^{\text {th }}, 2013$; revised July $15^{\text {th }}, 2013$; accepted August $10^{\text {th }}, 2013$

Copyright (C) 2013 Mandy A. Scott, Michael A. Persinger. This is an open access article distributed under the Creative Commons Attribution License, which permits unrestricted use, distribution, and reproduction in any medium, provided the original work is properly cited.

\begin{abstract}
Human cerebral systems are immersed in the earth's magnetic field. To be consistent with the results of several correlational studies, we found that the most accurate detection of information at $50 \mathrm{~m}$ occurred when the geomagnetic activity was $\sim 5 \mathrm{nT}$. The corresponding magnetic energy within the cerebral volume is equivalent to approximately 3 million bits of Landauer Limit quantum which is equivalent to low resolution photographs. Non-linear analyses indicated that the induced electric fields from the typical time variation of geomagnetic intensity converged with the Adey voltages for the threshold for background entropy. The relevance of signal/noise ratios and the recent evidence indicate that imagery and cognition may actually reflect fields of biophotons within a fixed volume, which indicates that a natural processing system may be occurring under very specific conditions that involves detection of recondite information at a distance.
\end{abstract}

Keywords: Geomagnetic Activity; Landauer Limit; Adey Voltages; Cerebral Energies; Magnetic Energy Bit Equivalents

\section{Introduction}

Of the myriad of physical events that penetrate cerebral space per second only an extremely small subset appear to evoke intracerebral changes that result in measureable responses [1]. Although traditional approaches to information processing within cerebral tissue assume transduction through sensory systems, the brain and the body also have the potential to behave as loci for energetic transformations. Recent quantitative electroencephalographic measurements have demonstrated specific alterations in frequency bands and amplitudes $\left(\sim \mu \mathrm{V}^{2} \cdot \mathrm{Hz}^{-1}\right)$ in the human brain activity during the geomagnetic activity $[2,3]$. The quantitative changes represented by these cerebral cortical indicators are in the same order of magnitude as the energy density within the cerebral volume associated with geomagnetic activity [3,4] which is within the range of tens of nanoTeslas (nT).

The measureable responsiveness of the cerebral activity to changes in geomagnetic activity suggests that human brains, which are immersed in the "static" global geo- magnetic field whose average is $\sim 50,000 \mathrm{nT}$, have

${ }^{*}$ Corresponding author. the potential to respond to distal information contained within it. Several studies have indicated that the right hemi-sphere of the human brain is more responsive to subtle changes in geomagnetic activity $[5,6]$. The right hemisphere, which is particularly activated during dreaming, is more specialized than the left for spatial processing, emotional interpretations, and pictorial rather than verbal classes of information processing [7]. Because left hemispheric processing dominates language and conscious experiences, the person may not necessarily be aware of responding to stimuli mediated by the right hemisphere [8]. However the "information" could still be processed and affect subordinate neural systems that control the body's approximately 10 trillion cells.

Operationally a signal is a stimulus whose information affects a response. That the human brain under specific conditions could discern the spatial and emotional information within distal stimuli was investigated from the engineering perspective of signal and information processing by Puthoff and Targ [9] at Stanford Research Institute during the 1970s. Over several years they found that some subjects could draw distant objects of which the experients were unaware and that these drawings 
were of sufficient detail to be accurately identified by judges not involved with the experiment. Like the seminal research of Krippner and his colleagues [10] during the 1960s, the accuracies displayed marked day-to-day variation and were not easily described by contemporary biophysical or information-based models.

Persinger and Krippner [11], employing the concept of signal/noise ratios for qualitative information processing found that the accuracy for the detection of distal stimuli was most conspicuous during days or nights when the global geomagnetic was most quiet at the time of the experience. In this instance the strongest congruence between the drawings or descriptions and the actual stimuli occurred when the global geomagnetic activity, as inferred by the aa (average antipodal) index, was less than $\sim 10 \mathrm{nT}$ and more likely within about the $5 \mathrm{nT}$ range. Similar values had been obtained from the analyses of hundreds of spontaneous cases that had been reported for more than a century [12].

Although historically these phenomena have been considered peripheral and at most probationary as legitimate events, we reasoned that the persistence of these episodes of putative detection of distant information implied a degree of reliability that could be examined routinely in experimental settings. If these phenomena were valid then they should be subject to routine replication and the contingencies under which they occured should be compatible with physical mechanisms. Here, as a component of our study of global, natural information systems, we present quantitative evidence by calculation and experimental evidence that the human brain has the capacity to detect information at a distance and that the process is most accurate when there is a narrow band of geomagnetic activity that matches the physical chemical restraints [13] that define the boundaries of brain activity.

\section{Model and Quantitative Solutions}

The energy that is lost to entropy or converges with another computation associated with 1 bit of information $(0,1)$ according to Landauer's limit is $(\mathrm{kT}) \ln 2$, where $\mathrm{T}$ is absolute temperature and $\mathrm{k}$ is Boltzmann's constant $\left(1.38 \cdot 10^{-23} \mathrm{~J} \cdot \mathrm{T}^{-1}\right)$. Assuming the median temperature of the human brain $\left(310^{\circ} \mathrm{K}\right)$ this quantum is $2.95 \cdot 10^{-21} \mathrm{~J}$. A text contains about $10^{2}$ bits ( 80 bits or 10 bytes), a simple drawing contains $\sim 10^{3}$ bits and a low resolution photograph involves $\sim 10^{6}$ bits. The amount of magnetic energy represented within space can be estimated by

$$
J=B^{2}(2 \cdot 4 \pi \mu)^{-1} \mathrm{~m}^{3}
$$

where $B$ is the net strength of the change in the field, $\mu$ is magnetic susceptibility and $\mathrm{m}^{3}$ is volume. For a net change of $5 \mathrm{nT}\left(5 \cdot 10^{-9} \mathrm{~T}\right)$, the optimal peak to peak variation associated with historical examples of detecting information at a distance, the energy represented within a human cerebrum of $\sim 10^{-3} \cdot \mathrm{m}^{3}$ would be $\sim 10^{-14} \mathrm{~J}$. In principle, if there are $2.95 \cdot 10^{-21} \mathrm{~J}$ per bit or unit convergence per "computation", then this amount of intracerebral magnetic energy could accommodate about $3 \cdot 10^{6}$ bits.

However, according to this equation a stronger field should allow more energy representation and hence more information to be accessed potentially. The narrow band around $5 \mathrm{nT}$ suggests a mechanism that, like resonance for frequency, occupies a discrete amplitude. One of the most likely candidates is the boundary for thermal limitations. According to the innovative solution by Adey [14], the threshold voltage required for an applied field to exceed background thermal agitation is:

$$
V^{2}=4 k T b \Omega
$$

where $k$ is the Boltzmann constant and $T$ is physiological temperature $\left(\sim 310^{\circ} \mathrm{K}\right), b$ is the frequency band, and $\Omega$ is resistance of the extracellular fluid or $2 \Omega \mathrm{m}$ [15]. If we assume a frequency "band" for natural geomagnetic variations in the order of $5 \mathrm{nT}$ is $10^{-2} \mathrm{~Hz}$, the solution is $\sim 8 \cdot 10^{-12} \mathrm{~V}$. This solution, that is congruent with the electrical field potential represented within the cross sectional area of the body $\left(\sim 0.1 \mathrm{~m}^{2}\right)$ from a change in $5 \cdot 10^{-9}$ $\mathrm{T}$ at $10^{-2} \mathrm{~Hz}$, would be $\sim 5 \cdot 10^{-12} \mathrm{~V}$ which is the same order of magnitude. Clearly this does not prove congruence or even validity. However it suggests that physical mechanisms could be accessed to test the concept. The magnetic energy $\left(10^{-14} \mathrm{~J}\right)$ associated with a $5 \mathrm{nT}$ field divided by Adey's voltage for the threshold where the electric field induced by the applied field converges with the temperature of the Landauer limit for energy lost (or gained) with 1 bit of information results in about $10^{-2}$ Coulombs $(\mathrm{A} \cdot \mathrm{s})$. This is equivalent to about 10 to 100 $\mathrm{mA} \cdot \mathrm{s}^{-1}$ which is well within the empirical range of intrinsic peak currents within the brain and body.

The second quantitative solution that supports the validity of the approach to accommodate access of information at a distance is the diffusivity. It can be derived from voltage divided by magnetic field strength. Assuming the typical $\sim 5 \cdot 10^{-3} \mathrm{~V}$ dc potentials between $\mathrm{cm}$ separated loci on the cerebral cortical surface and the net change of $\sim 5 \mathrm{nT}$ in static magnetic field displacement the ratio results in $\sim 10^{6} \mathrm{~m}^{2} \cdot \mathrm{s}^{-1}$. This is remarkably concordant with magnetic diffusivity [16]

$$
\mu^{-1} \sigma
$$

which is the product of the inverse of magnetic permeability and conductivity. With $2.1 \mathrm{~S} \cdot \mathrm{m}^{-1}$ for physiological saline and inverse $\mu=7.96 \cdot 10^{5} \mathrm{~A}^{2} \cdot \mathrm{N}^{-1}$ the diffusivity is $\sim 1.7 \cdot 10^{6} \mathrm{~m}^{2} \cdot \mathrm{s}^{-1}$. Given the range in actual brain values and geomagnetic variations, this incidental congruence would be associated with sufficient velocity to accommodate the required rate of information transfer. The 
linear velocity $\left(1.3 \cdot 10^{3} \mathrm{~m} \cdot \mathrm{s}^{-1}\right)$ across classic distances within brain tissue, $4 \mathrm{~mm}$ for the cerebral cortices and 1 $\mu \mathrm{m}$ for the width of an average synapse, would result in frequencies around $0.3 \mathrm{MHz}$ and $1.3 \mathrm{GHz}$, respectively.

\section{Empirical Support for Geomagnetic Modulation of Distal Information}

The test of the concept is the strength of the correlation between the accuracy for distant information and the geomagnetic activity at the time rather than understanding the complete mechanism, per se. We employed the methods described by others [9]. We were very familiar with this process because it is a component of our research with identifying the source localization (LORETA or low resolution electromagnetic tomography) and quantitative electroencephalographic profiles of people while they engage in introspective processes $[17,18]$.

Once per week for 3 successive weeks, each of 14 subjects sat in a quiet room for about $20 \mathrm{~min}$. Each subject was tested singly. Every week each subject was first brought by an experimenter to a small locked area where the information would be placed. The subject was told only there would be an opaque letter size envelope containing a photograph placed there in a short while. The subject was then escorted to the quiet room about $50 \mathrm{~m}$ away. While the subject was habituating and relaxing, the experimenter left the quiet room and returned to the locked room where one envelope containing a photograph was placed. The photographs, selected by a professional journalist (not associated with the experiment), for their emotional, affective and unusual content, had been placed in the sealed envelopes by a second experimenter (again not involved with the experiment) several weeks previously. The experimenter then returned to the quiet room where the subject was sitting. During the ensuing 20 min period the subject drew or stated what he or she experienced as images of what might be within the picture in the envelope in the locked room. After the draw- ings and statements were completed by a subject he or she was escorted to the locked room and the picture was viewed in order to facilitate learning and reinforcement for subsequent trials.

At the end of the study which involved 42 different pictures (3 times 14 subjects) two groups of 5 judges who did not know which pictures and descriptions were related and had not been involved with the experiment were asked to judge accuracies. For each "trial" each judge was given 4 pictures and the descriptions (drawings and words) of the subject for one of the pictures. Each judge ranked the congruence between the subject's description and each of the pictures. A rank of 1 indicated most related while a rank of 4 meant least related. The mean rank for 5 judges for the actual picture that was present when the subject described his or her experience was employed as the score. A score of 1 meant the judges all agreed that the description best fit the actual stimulus picture of the 4 pictures present. A score of 4 meant that there was no similarity. Scores in between were gradations of the two extremes.

The global geomagnetic activity $\left(\mathrm{K}_{\mathrm{p}}\right)$ was obtained for three hour increments before, during, and after the trials on any given day for all 42 measurements. In addition the mean $\mathrm{K}$ value for the day as well as the standard deviation of the 8 daily $\mathrm{K}$ values, as an indicator of "noise", were obtained. For comparison the A value, another type of geomagnetic daily indicator was obtained. These values were correlated (Pearson $r$ and Spearman rho) with the mean scores for accuracy of acquiring distant information. Table 1 shows the correlations for each of the three weeks. The significant positive correlations between accuracy and geomagnetic activity occurred during the time of the trials but not before or afterwards. This indicates that the geomagnetic association was specific to the period associated with the information access. The positive correlation indicated that the stronger the geomagnetic activity the lower the accuracy of the in-

Table 1. Parametric (r) and nonparametric (rho) correlation coefficients between accuracy scores for experiencing information at a distance and various global geomagnetic measures during the whole day (daily A) and proximal hours (3-hr K values) of the experiences. ${ }^{*} \mathrm{p}<0.05 ;{ }^{*}$ p $<0.01$.

\begin{tabular}{|c|c|c|c|c|c|c|c|c|}
\hline & \multicolumn{2}{|c|}{ Week 1} & \multicolumn{2}{|c|}{ Week 2} & \multicolumn{2}{|c|}{ Week 3} & \multicolumn{2}{|c|}{ Total } \\
\hline & $\mathrm{r}$ & rho & $\mathrm{r}$ & rho & r & rho & r & rho \\
\hline 3-hour during & 0.25 & 0.26 & $0.54^{*}$ & 0.36 & $0.64^{*}$ & $0.58^{*}$ & 0.22 & 0.22 \\
\hline 3-hour after & 0.25 & 0.25 & 0.21 & 0.31 & 0.10 & 0.27 & -0.09 & 0.01 \\
\hline Mean K values & 0.35 & 0.38 & $0.59^{*}$ & 0.50 & 0.52 & $0.55^{*}$ & 0.12 & 0.19 \\
\hline $\mathrm{SD}$ of $\mathrm{K}$ values & $0.80^{* *}$ & $0.79^{* *}$ & 0.22 & 0.37 & 0.34 & 0.38 & 0.14 & 0.30 \\
\hline Daily A value & 0.49 & $0.62^{*}$ & 0.36 & 0.36 & 0.20 & 0.18 & 0.02 & 0.15 \\
\hline
\end{tabular}


formation as indicated by the average score of five independent judges. The first obvious result was that the strength of association between the accuracy and the lower geomagnetic activity at the time of the acquisition of information increased over trials. However the average daily geomagnetic activity was not correlated significantly suggesting that the specific activity around the time of experiences was the critical factor. For the first week only there was a strong correlation between the increased variability ( $\mathrm{SD}$ of $\mathrm{K}$ value) in the $\mathrm{K}$ values around the time of the experience and the diminished accuracy.

Figure 1 shows the actual scatter for the relationship between the accuracy of the information drawn or described and the geomagnetic activity during this interval for the third session. $\mathrm{A} \mathrm{K}_{\mathrm{p}}$ value of 0 indicates $<5 \mathrm{nT}$. A score of 1 indicates that the information was matched by all judges as a first choice with the picture that was actually present.

\section{Discussion}

Cerebral networks can be conceived as natural loci of information processing from external and internal sources. The influence of signal-to-noise ratios is known to be one of the reasons that subtle afferent patterns to central processing regions within the cerebrum, even from smooth muscle, are masked by the more potent electromagnetic signals from skeletal muscle. Recent developments [19] in neuroscience have shown convincingly that portions of the cerebrum, particularly within the right hemisphere, respond to changes in subtle stimuli such as grammatical format, even though there is no "awareness". A similar process involves blind sight [20] for proximal visual stimuli.

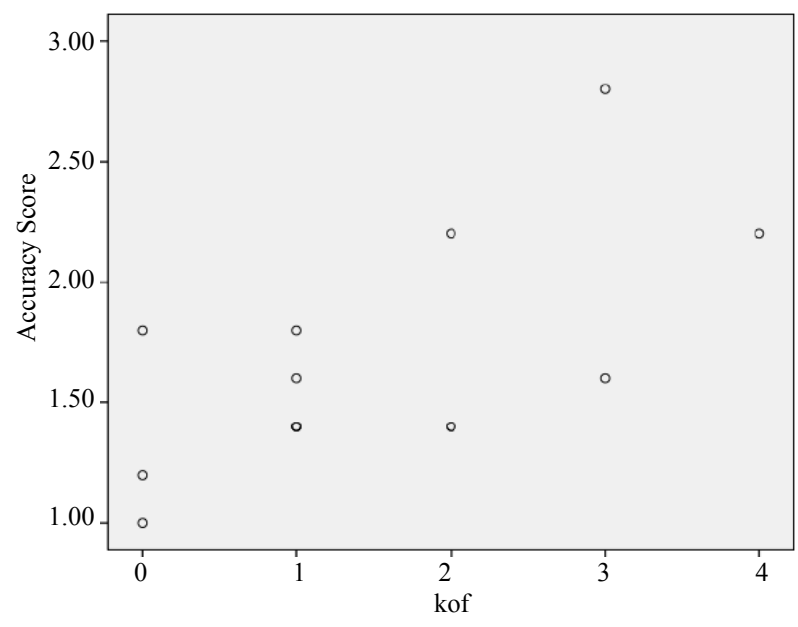

Figure 1. Accuracy score (lower score indicates greater congruency between target picture and experiences as ranked by independent judges) and the geomagnetic activity (kof) during the time of the information accessing.
That information from great distances can affect the brain through transduction of mechanical or photon energies to auditory and visual experiences defines the causal world for perception. However the actual physical sources responsible for the complexity of the fleeting images, feelings, and ideas that comprise the field of subjective experience and its phenomenology still remain incomplete. Explanations usually refer to unspecified local phenomena within the cerebrum. What is also clear is that as the subjects became more experienced with the process for responding to images and ideas concerning information at a distance the more the accuracy was associated with geomagnetic activity. The lowest geomagnetic activity at the time of the experiences was associated with the most congruence between those experiences (images and ideas) and the actual stimuli.

The results of this and other [9,10] studies indicate there could be a general class of information within the environment whose probability of detection is affected by the magnitude of geomagnetic activity which can be viewed as a form of "noise" that diminishes the signalto-noise ratio between the narrow physical chemical constraints that define cerebral activity and the natural world. This association would suggest that the information is strongly coupled to physical phenomena associated with the static geomagnetic field. Because all human brains are normally immersed in this field, theoretically they would have the capacity to access this information under optimal conditions. According to the original ideas of Bokkon [21] both analogue and digital information within brain space are strongly coupled with the quantum vacuum energies. He hypothesized that virtual particles, involved with vacuum fluctuation energies, and macroscopically reflected in Casimir-effects, may be a basis for information representation with brain space constituting "unconscious" experiences. With learning and reinforcement this information can approach a "critical mass" and be correlated with conscious experience. We think it is relevant that the Casimir forces and energies applied to the geometry of the synapse overlaps with the hydrogen frequency line $(1.42 \mathrm{GHz})$ which is prevalent throughout the universe.

According to our approach the optimal conditions occur within a non-linear system where the intensity of electric fields from background geomagnetic activity must overlap with the band-width dependent voltages determined by thermal limits within the human brain. The amount of energy within that narrow band would not be extreme but would be within the range that could accommodate the required bits to produce images and gestalts. The concept first developed by Bokkon [22] that thoughts and images are actually fields of biophotons which are generated by neuronal activity within the cerebral volume has been supported by both quantitative [23] 
and experimental data [24]. The involvement of photons, particularly in light of the new properties that emerge if they exhibit a discernable rest mass, indicates they may reflect a process that mediates the information that involves wider temporal frames or engages a longer "specious present" for inclusion.

\section{Conclusion}

Multiple correlational and experimental studies have shown weak but persistent evidence that under specific conditions the human brain can detect information at a distance through apparently non-traditional systems. This replication of an inverse correlation between global geomagnetic activity and accuracy of images of actual stimuli reported by more than a dozen subjects replicated these data. What is now clear is that the energy within the cerebral volume from the change in ambient magnetic fields is equivalent to more than a Megabit of Landauer Limit energy and that the electric field values from the ambient fields and the thermal thresholds converge. Like the first detection of Marconi's "S" under the optimal conditions of signal transmission that were based upon natural phenomena during the last century, our calculations and data suggest that employing a signal and information processing approach could be revealed for discovering another "recondite" mode of transferring information.

\section{Acknowledgements}

Thanks to Professor Blake Dotta for his technical contributions. The concepts in this manuscript are dedicated to the memory of Dr. William Roll.

\section{REFERENCES}

[1] T. Norretranders, "The User Illusion," Penguin, New York, 1991.

[2] E. S. Babayev and A. A. Alahverdiyeva, "Effects of Geomagnetic Activity Variations on the Physiological and Psychological State of Functionally Healthy Humans: Some Results from Azerbaijani Studies," Advances in Space Research, Vol. 40, No. 12, 2007, pp. 1941-1951. doi:10.1016/j.asr.2007.02.099

[3] B. P. Mulligan, M. D. Hunter and M. A. Persinger, "Effects of Geomagnetic Activity and Atmospheric Power variations on Quantitative Measures of Brain Activity: Replication of the Azerbaijani Studies," Advances in Space Research, Vol. 45, No. 7, 2010, pp. 940-948. doi:10.1016/j.asr.2009.12.008

[4] B. P. Mulligan and M. A. Persinger, "Experimental Simulation of the Effects of Sudden Increases in Geomagnetic Activity upon Quantitative Measures of Human Brain Activity: Validation of Correlational Studies," Neuroscience Letters, Vol. 516, No. 1, 2012, pp. 54-56. doi:10.1016/j.neulet.2012.03.054
[5] J. C. Booth, S. A. Koren and M. A. Persinger, "Increased Feelings of the Sensed Presence and Increased Geomagnetic Activity at the Time of the Experience during Exposures to Transcerebral Weak Complex Magnetic Fields," International Journal of Neuroscience, Vol. 115, No. 7, 2005, pp. 1039-1065. doi:10.1080/00207450590901521

[6] N. K. Belisheva, A. N. Popov, N. V. Petukhova, L. P. Pavlova, K. S. Osipov, S. E. Tkachenko and T. I. Baranova, "Quantitative and Qualitative Evaluations of the Effect of Geomagnetic Field Variations on the Functional State of the Human Brain," Biophysics, Vol. 40, 1995, pp. 1007-1014.

[7] D. Ottoson, "Duality and Unity of the Brain: Unified Functioning and Specialization of the Hemispheres," Plenum Press, New York, 1987.

[8] J. A. Hobson, "The Dreaming Brain," Basic Books, New York, 1988.

[9] H. E. Puthoff and R. Targ, "A Perceptual Channel for Information Transfer over Kilometer Distances: Historical Perspective and Recent Research," Proceedings of the IEEE, Vol. 64, No. 3, 1976, pp. 329-354.

[10] S. Krippner and L. George, "Psi Phenomena as Related to Altered States of Consciousness," In: B. B. Wolman and M. Ullman, Eds., Handbook of States of Consciousness, Van Nosrtrand Reinhold, New York, 1986, pp. 325-355.

[11] M A. Persinger and S. Krippner, "Dream ESP Experiments and Geomagnetic Activity," Journal of the American Society for Psychical Research, Vol. 83, No. 2, 1981, pp. 101-116.

[12] M. A. Persinger, "Spontaneous 'Telepathic' Experiences from Phantasms of the Living and Low Geomagnetic Activity," Journal of the American Society for Psychical Research, Vol. 81, 1987, pp. 23-26.

[13] M. A. Persinger, "Solutions for Real Values in Minkowski Four-Dimensional Space May Link Macro and Micro-Quantum Processes in the Brain," Neuroscience and Biobehavioral Reviews, Vol. 36, No. 10, 2012, pp. 2334-2338. doi:10.1016/j.neubiorev.2012.09.011

[14] W. R. Adey, "Tissue Interaction with Nonionizing Electromagnetic Fields," Physiological Reviews, Vol. 61, No. 2, 1981, pp. 435-514.

[15] F. A. Barnes, "Interaction of dc Electric Fields with Living Matter," In: C. Pollack and E. Postow, Eds., Handbook of Biological Effects of Electromagnetic Fields, CRC Press, Boston, 2000, pp. 99-120.

[16] G. Ryskin, "Secular Variation of the Earth's Magnetic Field: Induced by Ocean Flow?" New Journal of Physics, Vol. 11, 2009, pp. 1-23. doi:10.1088/1367-2630/11/6/063015

[17] C. F. Lavallee and M. A. Persinger, "A LORETA Study of Mental Time Travel: Similar and Distinct Electrophysiological Correlates of Re-Experiencing Past Events and Pre-Experiencing Future Events," Consciousness and Cognition, Vol. 19, No. 4, 2010, pp. 1037-1044. doi:10.1016/j.concog.2010.06.008

[18] M. A. Persinger and K. S. Saroka, "Protracted Parahippocampal Activity Associated with Sean Harribance," In- 
ternational Journal of Yoga, Vol. 5, No. 2, 2012, pp. 140145.

[19] G. S. Berns, J. D. Cohen and M. A. Mintun, "Brain Regions Responsive to Novelty in the Absence of Awareness," Science, Vol. 276, No. 5316, 1997, pp. 1272-1275. doi:10.1126/science.276.5316.1272

[20] R. Fendrich, C. M. Wessinger and M. S. Gazzaniga, "Residual Vision in Scotoma: Implications for Blindsight," Science, Vol. 258, No. 5087, 1992, pp. 1489-1491. doi:10.1126/science.1439839

[21] I. Bokkon, "Creative Information," Journal of Biological Systems, Vol. 11, No. 1, 2003, pp. 1-17. doi:10.1142/S0218339003000622

[22] I. Bokkon, "Dreams and Neuroholography: An Interdisciplinary Interpretation of the Development of Homeotherms in Evolution," Sleep and Hypnosis, Vol. 7, 2005, pp. 61-76.

[23] I. Bokkon, V. Salari, J. A. Tuszynski and I Antal, "Estimation of the Numbers of Biophotons Involved in Visual Perception of a Single-Object Image: Biophoton Intensity Can be Considered Higher Inside Cells Than Outside," Journal of Photochemistry and Photobiology B, Vol. 100, No.3, 2010, pp. 160-166. doi:10.1016/j.jphotobiol.2010.06.001

[24] B. T. Dotta, K. S. Saroka and M. A. Persinger, "Increased Photon Emissions from the Head While Imagining Light in the Dark Is Correlated with Changes in Electroencephalographic Power: Support for Bokkon's Biophoton Hypothesis," Neuroscience Letters, Vol. 513, No. 2, 2012, pp. 151-154. doi:10.1016/j.neulet.2012.02.021 